Research Article

\title{
Efficacy and Safety of Sipjeondaebo-Tang (Shi-Quan-Da-Bu-Tang) for Chronic Fatigue Syndrome: Study Protocol for a Multicenter, Randomized, Double-Blind, Placebo-Controlled Trial
}

\author{
Hyunjoo Oh $\mathbb{D},{ }^{1,2}$ Dong-Hyun Nam $\mathbb{D}^{3},{ }^{3}$ and Minwoo Hwang $\mathbb{D}^{4}$ \\ ${ }^{1}$ Department of Clinical Korean Medicine, Graduate School, Kyung Hee University, Seoul 02447, Republic of Korea \\ ${ }^{2}$ Medical \& Oriental Comprehensive Healthcare Center, Kyung Hee University Hospital, Seoul 02447, Republic of Korea \\ ${ }^{3}$ Department of Biofunctional Medicine and Diagnosis, College of Korean Medicine, Sangji University, Wonju 26339, \\ Republic of Korea \\ ${ }^{4}$ Department of Sasang Constitutional Medicine, College of Korean Medicine, Kyung Hee University, Seoul 02447, \\ Republic of Korea
}

Correspondence should be addressed to Minwoo Hwang; azure92@gmail.com

Received 30 March 2020; Revised 1 September 2020; Accepted 23 October 2020; Published 6 November 2020

Academic Editor: Ciara Hughes

Copyright (C) 2020 Hyunjoo Oh et al. This is an open access article distributed under the Creative Commons Attribution License, which permits unrestricted use, distribution, and reproduction in any medium, provided the original work is properly cited.

Background. Sipjeondaebo-tang (SDT), also known as Shi-Quan-Da-Bu-Tang, is a treatment for both qi and blood deficiency syndromes in traditional Korean medicine. It is also used to treat chronic fatigue syndrome (CFS) in Korea. Herein, we present the protocol for a study to assess the efficacy and safety of SDT for treating CFS. Methods. This will be a multicenter, randomized, double-blind, controlled trial with two parallel-treatment arms: an SDT group and a placebo group. Ninety-six patients with CFS aged between 19 and 65 years will be recruited from two hospitals in Korea. Participants will be randomly allocated at a ratio of $1: 1$ between the two groups. Participants will receive $3 \mathrm{~g}$ doses of SDT or placebo thrice daily for 8 weeks. Follow-up evaluations will be performed for 4-6 weeks after the drug administration period. The primary outcome will be the rating of participants' fatigue symptoms using the Checklist Individual Strength questionnaire. Outcomes will be assessed at baseline, week 4, and week 8 , as well as during follow-up. An efficacy evaluation and safety assessment will be performed. This study will be based on the Consolidated Standards of Reporting Trials (CONSORT) guidelines and the Standard Protocol Items: Recommendations for Interventional Trials (SPIRIT) 2013 statement. This protocol and informed consent guidelines were reviewed and approved by the institutional review board of Kyung Hee University Korean Medicine Hospital at Gangdong in the Republic of Korea (KHNMCOH 2017-06-004-001). The protocol was registered with the Clinical Research Information Service. Written informed consent will be obtained from all study participants prior to enrollment in the study. Results will be published in a peer-reviewed journal and presented at a scientific conference. Discussion. This study is expected to provide novel, accurate information regarding the 38 efficacy and safety of SDT for CFS in adults. Trial Registration. This trial is registered with https://cris.nih.go.kr; CRIS identifier (KCT0002684) registered on February 9, 2018.

\section{Introduction}

Chronic fatigue syndrome (CFS), which is synonymous with myalgic encephalomyelitis, is a condition characterized by pervasive fatigue (particularly after minimal exertion), chronic pain, and impaired concentration and memory [1]. It is assumed that CFS involves false fatigue alarms generated by predisposing and precipitating factors, which subsequently perpetuate stress responses [2]. There are no established biomarkers for CFS; therefore, the diagnosis must be based on a patient's description of the symptoms [3]. The most widely used diagnostic criteria are those published by the Centers for Disease Control and Prevention (CDC) in 1994. The diagnosis is made on the basis of 
prolonged (>6 months), unexplained, and incapacitating fatigue and fulfilling at least four of eight accompanying criteria [4]. The overall incidence rate of diagnosed CFS has been shown to range from 14.8 to 25.8 per 100,000 person-years, peaking in teenagers and adults in their thirties $[5,6]$. The prevalence of self-reported CFS has been reported to be $3.28 \%$ [7]. The prevalence of diagnosed CFS ranges from $0.083 \%$ to $2.3 \%$ in males and from $0.37 \%$ to $1.8 \%$ in females $[8,9]$. In Korea, the prevalence of chronic fatigue that is physically or psychologically explained is $8.4 \%$, whereas the prevalence of medically unexplained chronic fatigue, including CFS and idiopathic chronic fatigue, is $0.6 \%$ [10].

Currently, there is no cure or approved treatment for CFS. However, patients may find some symptom relief through nutritional supplements, exercise, rehabilitation programs, and cognitive behavioral therapy [11-14]. Intravenous injection of rituximab has been used to treat CFS in the past decade; however, a recent study showed that rituximab had no effect on CFS [15]. Research on herbal medications in East Asia has been conducted to find a curative treatment for CFS because some medications from the region are thought to be effective for fatigue and weariness. In previous studies, traditional Chinese medicine (TCM) has been proven effective for chronic fatigue [16-24]. However, according to a recent systematic review, previous studies evaluating the efficacy of TCM on CFS have not provided enough evidence or lacked methodological rigorousness to draw firm conclusions $[25,26]$. In traditional Korean medicine (TKM), there have been some randomized controlled trials (RCTs) evaluating the efficacy of some herbal medications on CFS [27-29]. However, no study up to now has focused on the efficacy of traditional herbal formulas used clinically for patients with chronic fatigue. Therefore, well-designed RCTs evaluating the efficacy of herbal TCM and TKM medications for CFS are needed.

Sipjeondaebo-tang (SDT), also called Shi-Quan-Da-BuTang in China and Juzen-taiho-to in Japan [30], is one of the most popular TKM formulas for the treatment of chronic fatigue. It is approved by the Ministry of Food and Drug Safety to treat weakness after illness, anorexia, abnormal sweating, peripheral coldness, and anemia [31]. Previous clinical trials have shown that it alleviates hematotoxicity, activates an anti-inflammatory effect, and promotes hematopoiesis [32-36]. In TKM, the main contributors to CFS are considered to be qi and blood deficiencies; if the flow of blood or qi is not smooth, fatigue may occur. SDT is used to treat chronic illnesses, including CFS, caused by both qi and blood deficiencies by balancing yin and yang. This is known to restore the deterioration of physiological function and activate immune function [37]. Although SDT is generally used to treat CFS clinically, no RCT has examined the efficacy of SDT for CFS. Therefore, in this study, we aim to assess the efficacy and safety of SDT for the treatment of CFS.

\section{Materials and Methods}

2.1. Study Design and Setting. This clinical trial will be conducted as a randomized, double-blind, placebo- controlled, parallel-group, multicenter study within 12-14 weeks at the Kyung Hee University Korean Medicine Hospital at Gangdong and the Woosuk University Korean Medicine Medical Center, both of which are clinical centers in the Republic of Korea. The study was designed in accordance with the Declaration of Helsinki and the Guidelines for Good Clinical Practice.

The principal investigator or researcher will obtain written informed consent from all participants after they have received a sufficient explanation of the study and made a thoughtful, voluntary decision. Each subject will be screened up to 7 days prior to randomization to avoid changes in subjects' health conditions. Once participants pass the eligibility assessment and receive a trial subject identifier, they will be randomly assigned to either the treatment group or the control group in a $1: 1$ ratio. The treatment group will be prescribed SDT, and the control group will be prescribed a placebo for 8 weeks after attending an educational session regarding the clinical trial. Participants will visit the study site every 4 weeks during the treatment period to allow researchers to evaluate their compliance and the efficacy and safety of the treatment. A follow-up will be completed by phone 4-6 weeks after the completion of the drug administration to evaluate safety. Any medication not consumed by the participants will be returned at visits 2 and 3 to allow us to calculate drug compliance. The evaluation of participants and analysis of the results will be performed by professionals blinded to the group allocation. Figure 1 shows a schematic flow diagram of the study. The schedule of enrollment, intervention, and assessment is presented in Table 1 .

2.2. Patient Involvement. Patients were not involved in the research design process. The study is expected to run from May 2018 to December 2020.

\subsection{Participants}

2.3.1. Inclusion Criteria. A total of 96 patients will be recruited via outdoor advertisements or from the outpatient clinics of the two hospitals. Participants must meet all of the following criteria:

(1) Male or female older than 19 years of age

(2) Those with 76 points or more on the Korean Checklist Individual Strength questionnaire (CIS) [38, 39].

(3) Those who fulfill all of the following items based on the CFS diagnosis criteria published by the CDC [4].

(a) The individual who has severe chronic fatigue for 6 or more consecutive months that is not due to ongoing exertion or other medical conditions associated with fatigue (these other conditions need to be ruled out by a doctor after diagnostic tests have been conducted)

(b) The fatigue that significantly interferes with daily activities and work 


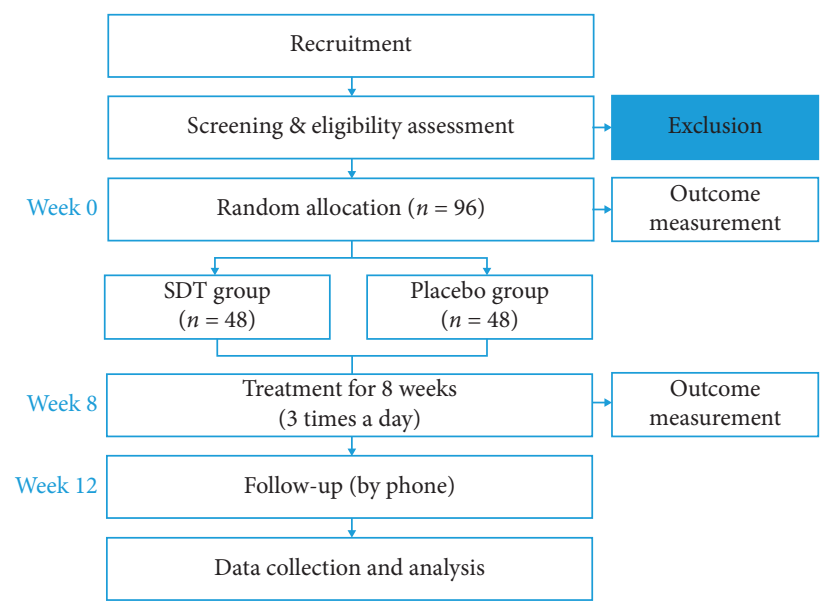

Figure 1: Flowchart of the study. Abbreviation: SDT, Sipjeondaebo-tang.

TABLE 1: Schedule of enrollment, intervention, and assessments.

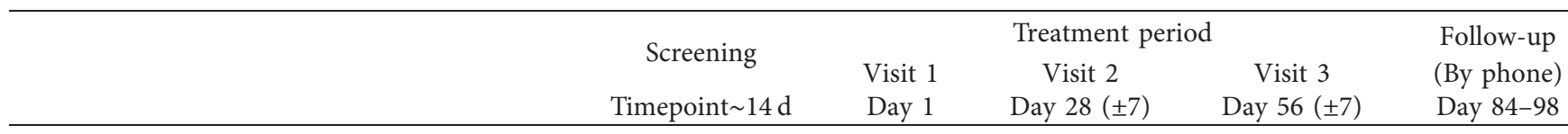

Patients

Informed consent

Demographics

Medical history

Physical examinations

Vital signs

Laboratory examination

Pregnancy test

HRV measurement

Body composition measurement

Confirmation of inclusion/exclusion criteria

Randomization

Intervention

Medication

Trial evaluation

Investigation of drug compliance

Investigation of concomitant medication

Adverse reactions

\section{Outcomes}

CIS

VAS for fatigue, happiness, and CDC criteria

FSS

EQ-5D-5L

PSQI-K

ChFI

QBYY-Q

Original symptom questionnaire

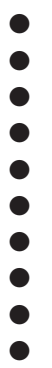

$\bullet$

Abbreviations: HRV, heart rate variability; CIS, Checklist Individual Strength; VAS, visual analogue scale; CDC, center for disease control and prevention; FSS, fatigue severity scale; EQ-5D-5L, EuroQol 5-dimensions, 5-level questionnaire; PSQI-K, the Korean version of Pittsburgh Sleep Quality Index; ChFI, Chalder fatigue scale; QBYY-Q, Qi blood Yin Yang deficiency questionnaire.

(c) The individual who concurrently has four or more of the following eight symptoms

(i) Postexertion malaise lasting more than 24 hours

(ii) Unrefreshing sleep (iii) Significant impairment of short-term memory or concentration

(iv) Muscle pain

(v) Multijoint pain without swelling or redness

(vi) Headaches of a new type, pattern, or severity 
(vii) Tender cervical or axillary lymph nodes

(viii) A sore throat that is frequent or recurring

2.3.2. Exclusion Criteria. Participants who meet any of the following conditions will not be able to participate in this study:

(1) Those with a medical history of hypersensitivity to the investigational drugs or any herbal ingredients

(2) Those who have or are suspected of having anorexia or bulimia

(3) Those with a body mass index greater than $45 \mathrm{~kg} / \mathrm{m}^{2}$

(4) Those who present with at least moderate levels of liver dysfunction (e.g., alanine aminotransferase and aspartate aminotransferase levels $>100 \mathrm{IU} / \mathrm{L}$ or serum bilirubin level $>3.0 \mathrm{mg} / \mathrm{dL}$ ) or who have hepatitis, liver cirrhosis, or fatty liver and need medical attention

(5) Those with signs of active hepatitis and whose $\mathrm{HBs} A \mathrm{~g}$ and $\mathrm{HCV}-\mathrm{Ab}$ rapid tests are positive

(6) Those with a positive HIV test

(7) Those who present with kidney dysfunction (creatinine level $>2.0 \mathrm{mg} / \mathrm{dL}$ ) or who have any renal disease requiring dialysis

(8) Those with systolic blood pressure $>180 \mathrm{mmHg}$ or mean diastolic blood pressure $>110 \mathrm{mmHg}$

(9) Patients with generalized edema

(10) Patients with unregulated diabetes mellitus (hemoglobin A1c > 9\%)

(11) Patients with a medical history of malignant tumors within the past 5 years

(12) Patients with tuberculosis, multiple sclerosis, hypothyroidism, or asthma currently uncontrolled

(13) Patients with major depressive disorder, bipolar affective disorder, schizophrenia, dementia, or delusional disorder

(14) Patients with clinically significant gastrointestinal disease that may affect drug absorption, distribution, metabolism, or excretion

(15) Patients with myocardial infarction, unstable angina pectoris, stroke, cerebral ischemic attack, heart failure, or uncontrolled arrhythmia, or those who have undergone a coronary revascularization procedure within the last 6 months

(16) Women who are pregnant (urine human chorionic gonadotropin test positive within 28 days) or lactating

(17) Those scheduled for exercise therapy during the trial period

(18) Those with a history of alcohol or drug abuse in the past year

(19) Those who have participated in other clinical trials within the past 3 months

(20) Those who are judged to be inappropriate for the clinical trial by the researchers because of other clinically significant medical or psychiatric findings

2.4. Participant Withdrawal Criteria. Participants who meet any of the following criteria will be removed from the trial:

(1) Those with acute reactions to the investigational drugs

(2) Those who have received prohibited medications or therapies

(3) Those who need surgery or inpatient treatment due to emergencies such as accidents or unexpected diseases

(4) Those who become pregnant during the trial period

(5) The subject or his or her representative who withdraws consent

(6) Occurrence of a serious adverse reaction

(7) Researchers' decision to terminate an individual's participation in the trial due to the judgment that the participant's continuation is inappropriate

Reasons for withdrawal will be documented in case report forms (CRFs), and data will be analyzed using the intention-to-treat (ITT) principle.

2.5. Randomization. Study patients who meet the eligibility criteria will be randomly assigned to a treatment group (SDT) or a control group (placebo) in a $1: 1$ ratio through hospital-stratified randomization. Randomization will be conducted by a statistician using a computer-generated random allocation sequence in a SAS system through a predefined block randomization list with random block sizes. Doctors will allocate a number in the order of random assignment to each participant. Allocation concealment will be ensured; the randomization code will be released after the end of the trial.

2.6. Blinding. Both researchers and participants will be blinded to the allocation. The randomization code number on the investigational drugs will be labeled by a staff member of the pharmaceutical company producing the investigational drugs. The labeled trial products will be administered and provided to each participant by independent research pharmacists in each hospital who are also blinded to the randomization. The researchers may reveal blinded information only in the event of urgent medical conditions such as serious adverse events or voluntary withdrawals, in accordance with the standard operating procedures (SOPs). Any unblinding will be documented.

2.7. Interventions. Participants will be prescribed SDT or a placebo at a dose of $3 \mathrm{~g}$ three times a day for 8 weeks. Each dose will be recommended to be taken before or between meals with warm water. Both SDT and the placebo will be manufactured at a factory belonging to Hanpoong Pharm \& Foods Co. in Seoul, Republic of Korea, certified for Good 
Manufacturing Practice. Granulated SDT extract contains $1.0 \mathrm{~g}$ each of Poria sclerotium, Cnidii rhizoma, Cinnamomi ramulus, Rehmanniae radix preparata, Astragali radix, Paeoniae radix, Atractylodis rhizoma alba, Ginseng radix $a l b a$, and Angelicae gigantis radix and $0.5 \mathrm{~g}$ of Glycyrrhizae radix. The SDT will be extracted from these raw materials and concentrated to $3 \mathrm{~g}$ per dose. The granulated placebo extract will contain $1.5 \mathrm{~g}$ of corn starch, $1.5 \mathrm{~g}$ of lactose, $0.05 \mathrm{~g}$ of citric acid, $0.06 \mathrm{~g}$ of caramel coloring, and $0.002 \mathrm{~g}$ of Ginseng radix alba herbal flavor per dose. Placebo pills will have a similar appearance, shape, weight, taste, and flavor as those of SDT.

During the trial, participants will be prohibited from receiving any other medications or therapies that may influence the study outcomes. These medications and therapies include antipsychotics, antidepressants, dementia drugs, systemic steroids, immunomodulators, immunosuppressants, transfusions, other herbal medicines, and exercise therapy. Short-term use (i.e., $\leq 5$ days) of nonsteroidal antiinflammatory drugs will be allowed, but such drugs must not be taken within the 3 days preceding the efficacy evaluation for SDT and the placebo treatment. Detailed lists of prohibited and allowed medications will be made available to the participants.

2.8. Data Collection and Management. Outcome measurements will be collected, and data on the identification of participants, allocation of random assignments, and health assessments will be written on the CRF by a certified clinical research coordinator (CRC). Data entry and coding of all identifying participant information will also be performed by the CRC. Research data will be permanently stored on the researcher's computer, and documents will be kept in secured locations for more than three years.

\subsection{Outcomes}

2.9.1. Primary Outcome Measurement. Participants with total CIS scores $\leq 76$ will be rated as "responders," and the percentage of participants assessed as "responders" will be defined as the response rate for fatigue. The change in the response rate for fatigue between visit 1 (i.e., baseline) and visits 2 and 3 (i.e., posttreatment assessments) will be used as the primary outcome measure.

2.9.2. Secondary Outcome Measurements. Changes in the scores of the CIS; Visual Analogue Scale (VAS) for fatigue, happiness, and $\mathrm{CDC}$ criteria; Fatigue Severity Scale; EuroQol 5-dimensions, 5-level questionnaire; Korean version of Pittsburgh Sleep Quality Index; Chalder Fatigue Scale; and qi blood Yin Yang deficiency questionnaire between visit 1 and visits 2 and 3 will be used as the secondary outcome measures. All questionnaires will be used in their Korean-validated form [40-48]. The VAS will represent the participant's current status on a scale ranging from $0 \mathrm{~cm}$ (no symptoms) to $10 \mathrm{~cm}$ (maximum symptoms).
2.9.3. Safety Outcome Measurement. For the safety assessment, participants' vital signs will be documented, and general examinations will be performed at every visit. The occurrence of any adverse events (AEs) will be checked at visits 2 and 3 and during follow-up.

2.9.4. Compliance Calculation. To calculate drug compliance, participants will be asked to return any remaining investigational drugs at visits 2 and 3 . The rate of compliance will be calculated as follows: compliance $(\%)=($ expected intake - remaining products/expected intake $) \times 100$.

2.10. AE Reporting. The researchers will report any AEs that occur after administration. All AEs will be recorded in the $\mathrm{CRF}$ and evaluated for causal relationships. If AEs occur, an independent data monitoring committee will decide whether the study should continue. If serious AEs occur, the researchers must notify the institutional review board within 24 hours.

2.11. Sample Size. Based on a previous study, a sample-size calculation was completed to estimate the minimal number of patients required to detect a clinically significant difference of $17 \%$ in the primary outcome measure [49]. It is assumed that the response rates at both week 4 and week 8 will be similar. Seventy-eight patients are required to detect such a difference with $80 \%$ certainty using a double-sided significance level of 5\%. Anticipating a dropout rate of $20 \%$, 96 participants are needed.

\subsection{Statistical Analyses}

2.12.1. Efficacy Assessment. Efficacy evaluation will be carried out on the basis of the full analysis set, with additional analysis performed on the per-protocol set. Adjustments will be made for missing data using the last-observation-carriedforward imputation method. Continuous variables will be reported as the mean \pm standard deviation if normally distributed or the median and interquartile range if not normally distributed, and nominal variables will be reported as absolute numbers and percentages. To assess the primary outcome, a superiority test will be conducted. To assess secondary outcomes, an independent two-sample $t$-test or Wilcoxon test will be conducted on continuous variables and a chi-square test or Fisher's exact test will be conducted on nominal variables. Statistical analysis will be undertaken using the ITT principle with a 95\% confidence interval. All analyses will be performed using SPSS (V.19.0; IBM, Chicago, IL, USA). Significance for all tests will be set at $P<0.05$ in a two-tailed test.

2.12.2. Safety Assessment. A safety assessment will be carried out on the safety set. The safety data will be stratified according to symptoms, and a chi-square test or Fisher's exact test will be conducted. 
2.13. Monitoring. Monitoring will be performed by the contracted research organization. All hospitals conducting trials will be monitored during trials in accordance with the SOPs.

\section{Discussion}

To the best of our knowledge, this study is the first that aims to provide accurate information regarding the efficacy of SDT for CFS in adults. Though it has limitations, such as the possibility of measurement bias (participant self-rating scales may exaggerate the severity of CFS) and the limitation of the race (all participants will be Korean), it will demonstrate the effect of traditional herbal medication on CFS. There is still a lack of research on the effect of medications treating CFS; this study will help identify and expand our knowledge of potential medications for CFS treatment.

\section{Abbreviations}

SDT: Sipjeondaebo-tang

CFS: Chronic fatigue syndrome

CDC: Centers for disease control and prevention

TCM: Traditional Chinese medicine

TKM: Traditional Korean medicine

RCT: Randomized controlled trials

CIS: Checklist Individual Strength questionnaire

CRF: Case report forms

ITT: Intention-to-treat

SOP: Standard operating procedures

CRC: Clinical research coordinator

VAS: Visual analogue scale

AEs: Adverse events.

\section{Data Availability}

Data and materials are available upon request from the corresponding author.

\section{Ethical Approval}

The protocol design is based on the Consolidated Standards of Reporting Trials (CONSORT) guidelines and the Standard Protocol Items: Recommendations for Interventional Trials (SPIRIT) 2013 statement [50]. The protocol and informed consent guidelines were reviewed and approved by the institutional review board of Kyung Hee University Korean Medicine Hospital at Gangdong in the Republic of Korea (KHNMCOH 2017-06-004-001). The protocol was registered on the Clinical Research Information Service, which is a registry in the World Health Organization Registry Network (KCT0002684). Important, unavoidable protocol modifications will be reported directly by the researchers to the institutional review board. Only the researchers and the requested statistician have access to the final dataset.

\section{Consent}

Written informed consent was obtained from all study participants prior to enrollment.

\section{Conflicts of Interest}

The authors declare that they have no conflicts of interest.

\section{Authors' Contributions}

$\mathrm{HO}$ drafted this manuscript. $\mathrm{HO}$ and $\mathrm{MH}$ participated in the conception and design of the trial, developed the criteria, and created the CRF. DHN and $\mathrm{MH}$ contributed to quality control. $\mathrm{MH}$ registered the study. $\mathrm{HO}$ and $\mathrm{DHN}$ designed the statistical analysis, sample-size calculation, and randomization. All authors read and approved the final manuscript.

\section{Acknowledgments}

This study was supported by the Traditional Korean Medicine Research \& Development Program funded by the Ministry of Health \& Welfare through the Korea Health Industry Development Institute (HB16C0055) and the Bio \& Medical Technology Development Program of the National Research Foundation funded by the Ministry of Science and Information \& Communication Technology (NRF2014M3A9D7045482). The funding parties were not involved in the design or management of the study; in the data collection, analysis, or interpretation; or in the writing of the manuscript.

\section{References}

[1] Institute of Medicine, Beyond Myalgic Encephalomyelitis/ Chronic Fatigue Syndrome: Redefining an Illness, The National Academies Press, Washington, DC, USA, 2015.

[2] V. B. Wyller, S. E. Reme, and T. E. Mollnes, "Chronic fatigue syndrome/myalgic encephalo-myelitis-pathophysiology, diagnosis and treatment," Tidsskr Nor Laegeforen, vol. 135, no. 23-24, pp. 2172-2175, 2015.

[3] E. Ur, P. D. White, and A. Grossman, "Hypothesis: cytokines may be activated to cause depressive illness and chronic fatigue syndrome," European Archives of Psychiatry and Clinical Neuroscience, vol. 241, no. 5, pp. 317-322, 1992.

[4] K. Fukuda, S. E. Straus, I. Hickie et al., "The chronic fatigue syndrome: a comprehensive approach to its definition and study," Annals of Internal Medicine, vol. 121, no. 12, pp. 953-959, 1994.

[5] S. M. Collin, I. J. Bakken, I. Nazareth, E. Crawley, and P. D. White, "Trends in the incidence of chronic fatigue syndrome and fibromyalgia in the UK, 2001-2013: a clinical practice research datalink study," Journal of the Royal Society of Medicine, vol. 110, no. 6, pp. 231-244, 2017.

[6] I. Bakken, K. Tveito, N. Gunnes et al., "Two age peaks in the incidence of chronic fatigue syndrome/myalgic encephalomyelitis: a population-based registry study from Norway 2008-2012," BioMed Central Medicine, vol. 12, no. 1, p. 167, 2014.

[7] S. Johnston, E. W. Brenu, D. Staines, and S. Marshall-Gradisnik, "The prevalence of chronic fatigue syndrome/myalgic encephalomyelitis: a meta-analysis," Clinical Epidemiology, vol. 5, pp. 105-110, 2013.

[8] M. Reyes, R. Nisenbaum, D. C. Hoaglin et al., "Prevalence and incidence of chronic fatigue syndrome in Wichita, Kansas," 
Archives of Internal Medicine, vol. 163, no. 13, pp. 1530-1536, 2003.

[9] J. N. Baraniuk, "Chronic fatigue syndrome prevalence is grossly overestimated using Oxford criteria compared to Centers for Disease Control (Fukuda) criteria in a U.S. population study," Fatigue: Biomedicine, Health \& Behavior, vol. 5, no. 4, pp. 215-230, 2017.

[10] C. H. Kim, H. C. Shin, and C. W. Won, "Prevalence of chronic fatigue and chronic fatigue syndrome in Korea: communitybased primary care study," Journal of Korean Medical Science, vol. 20, no. 4, pp. 529-534, 2005.

[11] J. Castro-Marrero, N. Sáez-Francàs, D. Santillo, and J. Alegre, "Treatment and management of chronic fatigue syndrome/ myalgic encephalomyelitis: all roads lead to Rome," British Journal of Pharmacology, vol. 174, no. 5, pp. 345-369, 2017.

[12] L. Larun, K. G. Brurberg, J. Odgaard-Jensen, and J. R. Price, "Exercise therapy for chronic fatigue syndrome," Cochrane Database System Reviews, vol. 10, no. 10, Article ID CD003200, 2019.

[13] R. R. Taylor, "Rehabilitation programs for individuals with chronic fatigue syndrome," Journal of Chronic Fatigue Syndrome, vol. 13, no. 1, pp. 41-55, 2006.

[14] J. R. Price, E. Mitchell, E. Tidy, and V. Hunot, "Cognitive behaviour therapy for chronic fatigue syndrome in adults," Cochrane Database System Reviews, vol. 3, no. 3, Article ID CD001027, 2008.

[15] Ø. Fluge, I. G. Rekeland, K. Lien et al., "B-lymphocyte depletion in patients with myalgic encephalomyelitis/chronic fatigue syndrome," Annals of Internal Medicine, vol. 170, no. 9, pp. 585-593, 2019.

[16] R. Zhang, J. Li, J. Chen, Z. J. Zhang, and Z. H. Guo, “Clinical observation of Shenqi recovery tang in treatment of chronic fatigue syndrome," Journal of Traditional Chinese Medical Sciences, vol. 11, p. 148, 2004.

[17] R. M. Yao and M. Y. Qiu, "Clinical observation of chronic fatigue syndrome treatment by Chinese herbal medicine in Hong Kong," Shanghai Journal of Traditional Chinese Medicine, vol. 39, pp. 12-13, 2005.

[18] J. Z. Wang, B. Fang, H. F. Zhang, and C. Y. Wu, "Clinical study of Fuzheng Jieyu prescription in treatment of chronic fatigue syndrome," Chinese Journal of Information on Traditional Chinese Medicine, vol. 14, pp. 75-76, 2007.

[19] B. Fang, J. Z. Wang, and H. F. Zhang, "Clinical observation of Xiaopiling particles in treatment of chronic fatigue syndrome," Chinese Journal of Integrated Traditional and Western Medicine, vol. 16, pp. 1622-1623, 2007.

[20] Y. Q. Fang, Y. L. Ren, and G. T. Wang, "Clinical observation of Fufang Shenqi ointment in treatment of chronic fatigue syndrome," Northwest Pharmaceutical Journal, vol. 23, pp. 389-390, 2008.

[21] Z. X. Zhang, L. L. Wu, and M. Chen, "Effect of lixu jieyu recipe in treating 75 patients with chronic fatigue syndrome," Zhongguo Zhong Xi Yi Jie He Za Zhi, vol. 29, no. 6, pp. 501-505, 2009.

[22] C. D. Li, Y. L. Chen, and L. Huang, "Clinical observation of soothing liver and activating spleen method in treatment of chronic fatigue syndrome," Liaoning Journal of Traditional Chinese Medicine, vol. 38, pp. 2037-2038, 2011.

[23] D. Xu, Y. X. Dong, and X. Q. Yang, "Effect observation of JiaweiNaoxinkang in treatment of chronic fatigue syndrome in 40 patients," Journal of Changchun University Traditional Chinese Medicine, vol. 29, pp. 281-282, 2013.

[24] Y. H. Pang and J. P. Liu, "Therapeutic effect of shengmai powder plus modified xiao powder for treatment of chronic fatigue syndrome," Journal of Guangzhou University of Traditional Chinese Medicine, vol. 30, pp. 316-319, 2013.

[25] Y.-Y. Wang, X.-X. Li, J.-P. Liu, H. Luo, L.-X. Ma, and T. Alraek, "Traditional Chinese medicine for chronic fatigue syndrome: a systematic review of randomized clinical trials," Complementary Therapies in Medicine, vol. 22, no. 4, pp. 826-833, 2014.

[26] D. Adams, T. Wu, X. Yang, S. Tai, and S. Vohra, "WITHDRAWN: traditional Chinese medicinal herbs for the treatment of idiopathic chronic fatigue and chronic fatigue syndrome," Cochrane Database System Reviews, vol. 10, no. 10, Article ID CD006348, 2018.

[27] J. H. Cho, C. K. Cho, J. W. Shin, J. Y. Son, W. Kang, and C. G. Son, "Myelophil, an extract mix of Astragali Radix and Salviae Radix, ameliorates chronic fatigue: a randomised, double-blind, controlled pilot study," Complementary Therapies in Medicine, vol. 17, no. 3, pp. 141-146, 2009.

[28] K. K. Lee, W. S. Choi, K. S. Yum et al., "Efficacy and safety of human placental extract solution on fatigue: a double-blind, randomized, placebo-controlled study," Evidence Based Complement Alternative Medicine, vol. 2012, Article ID 130875, 6 pages, 2012.

[29] H. G. Kim, J. H. Cho, S. R. Yoo et al., “Antifatigue effects of Panax ginseng C.A. Meyer: a randomised, double-blind, placebo-controlled trial," PLoS One, vol. 8, no. 4, Article ID e61271, 2013.

[30] C. Cheon, S. Kang, Y. Ko et al., "Sipjeondaebo-tang in patients with breast cancer with fatigue: a protocol for a pilot, randomized, double-blind, placebo-controlled, cross-over trial," The Open BioMed Journal, vol. 8, no. 7, Article ID e021242, 2018.

[31] Ministry of Food and Drug Safety, Online Medicine Library, Ministry of Food and Drug Safety, Cheongju, South Korea, 2019, https://nedrug.mfds.go.kr/pbp/CCBBB01/ getItemDetail?itemSeq=197900047.

[32] S.-M. Huang, L.-Y. Chien, C.-J. Tai, J.-F. Chiou, C.-S. Chen, and C.-J. Tai, "Effectiveness of 3-week intervention of Shi Quan $\mathrm{Da}$ Bu Tang for alleviating hematotoxicity among patients with breast carcinoma receiving chemotherapy," Integrative Cancer Therapies, vol. 12, no. 2, pp. 136-144, 2013.

[33] A. Chino, H. Sakurai, M.-K. Choo et al., "Juzentaihoto, a Kampo medicine, enhances IL-12 production by modulating Toll-like receptor 4 signaling pathways in murine peritoneal exudate macrophages," International Immunopharmacology, vol. 5, no. 5, pp. 871-882, 2005.

[34] H. Kiyohara, T. Nagai, K. Munakata et al., "Stimulating effect of Japanese herbal (kampo) medicine, hochuekkito on upper respiratory mucosal immune system," Evidence-Based Complementary and Alternative Medicine, vol. 3, no. 4, pp. 459$467,2006$.

[35] H. Nakamoto, T. Mimura, and N. Honda, "Orally administrated Juzen-taiho-to/TJ-48 ameliorates erythropoietin (rHuEPO)-resistant anemia in patients on hemodialysis," Hemodialysis International, vol. 12, no. S2, pp. S9-S14, 2008.

[36] Y. Kishida, T. Nishii, T. Inoue et al., "Juzentaihoto (TJ-48), a traditional Japanese herbal medicine, influences hemoglobin recovery during preoperative autologous blood donation and after hip surgery," International Journal of Clinical Pharmacology and Therapeutics, vol. 47, no. 12, pp. 716-721, 2009.

[37] S. Oh, C. Cheon, S. Park et al., "The analysis of the recent research trend of sipjeondabo-tang in Korea," Korean Society for Preventive Medicine, vol. 18, pp. 113-123, 2014.

[38] J. H. M. M. Vercoulen, C. M. A. Swanink, J. F. M. Fennis, J. M. D. Galama, J. W. M. van der Meer, and G. Bleijenberg, 
"Dimensional assessment of chronic fatigue syndrome," Journal of Psychosomatic Research, vol. 38, no. 5, pp. 383-392, 1994.

[39] Y. Kim and N. Cha, "Correlations among occupational stress, fatigue, and depression in call center employees in Seoul," Journal of Physical Theraphy Science, vol. 27, no. 1, pp. 3191-3194, 2015.

[40] L. B. Krupp, N. G. LaRocca, J. Muir-Nash, and A. D. Steinberg, "The fatigue severity scale," Archives of Neurology, vol. 46, no. 10, pp. 1121-1123, 1989.

[41] K. Kim and Y. S. Ha, "Factors influencing on fatigue in patients with epilepsy," Journal of Korean Epilepsy Society, vol. 13, no. 2, pp. 42-46, 2009.

[42] M. Herdman, C. Gudex, A. Lloyd et al., "Development and preliminary testing of the new five-level version of EQ-5D (EQ-5D-5L)," Quality of Life Research, vol. 20, no. 10, pp. 1727-1736, 2011.

[43] H. Lee, M. Jo, S. Choi et al., "Development and psychometric evaluation of measurement instrument for Korean healthrelated quality of life," Public Health Weekly Report (KCDC), vol. 9, no. 24, pp. 447-454, 2016.

[44] D. J. Buysse, C. F. Reynolds 3rd, T. H. Monk, S. R. Berman, and D. J. Kupfer, “The Pittsburgh Sleep Quality Index: a new instrument for psychiatric practice and research," Psychiatry Research, vol. 28, no. 2, pp. 193-213, 1989.

[45] S. I. Sohn, D. H. Kim, M. Y. Lee, and Y. W. Cho, "The reliability and validity of the Korean version of the Pittsburgh Sleep quality index," Sleep and Breathing, vol. 16, no. 3, pp. 803-812, 2012.

[46] T. Chalder, G. Berelowitz, T. Pawlikowska et al., "Development of a fatigue scale," Journal of Psychosomatic Research, vol. 37, no. 2, pp. 147-153, 1993.

[47] S. M. Oh, K. J. Min, and D. B. Park, "A study on the standardization of the hospital anxiety and depression scale for Koreans: a comparison of normal, depressed and anxious groups," Journal of Korean Neuropsychiatric Association, vol. 38, no. 2, pp. 289-296, 1999.

[48] J. Kim, B. Ku, and K. H. Kim, "Validation of the qi blood yin yang deficiency questionnaire on chronic fatigue," Chinese Medicine, vol. 11, p. 24, 2016.

[49] D. Blockmans, P. Persoons, B. Van Houdenhove, and H. Bobbaers, "Does methylphenidate reduce the symptoms of chronic fatigue syndrome?," The American Journal of Medicine, vol. 119, no. 2, p. 167, 2006.

[50] A.-W. Chan, J. M. Tetzlaff, D. G. Altman et al., "SPIRIT 2013 statement: defining standard protocol items for clinical trials," Annals of Internal Medicine, vol. 158, no. 3, pp. 200-207, 2013. 